\title{
NASA Langley Distributed Propulsion VTOL Tilt-Wing Aircraft Testing, Modeling, Simulation, Control, and Flight Test Development
}

\author{
Paul M. Rothhaar ${ }^{1}$, Patrick C. Murphy ${ }^{2}$, Barton J. Bacon ${ }^{3}$, Irene M. Gregory ${ }^{4}$, Jared A. Grauer ${ }^{5}$, \\ Ronald C. Busan ${ }^{6}$, and Mark A. Croom ${ }^{7}$ \\ NASA Langley Research Center, Hampton, Virginia, 23681
}

\begin{abstract}
Control of complex Vertical Take-Off and Landing (VTOL) aircraft traversing from hovering to wing born flight mode and back poses notoriously difficult modeling, simulation, control, and flight-testing challenges. This paper provides an overview of the techniques and advances required to develop the GL-10 tilt-wing, tilt-tail, long endurance, VTOL aircraft control system. The GL-10 prototype's unusual and complex configuration requires application of state-of-the-art techniques and some significant advances in wind tunnel infrastructure automation, efficient Design Of Experiments (DOE) tunnel test techniques, modeling, multi-body equations of motion, multi-body actuator models, simulation, control algorithm design, and flight test avionics, testing, and analysis. The following compendium surveys key disciplines required to develop an effective control system for this challenging vehicle in this on-going effort.
\end{abstract}

\section{Nomenclature}

$\begin{array}{llll}a_{k} & =\text { amplitude, deg } & \alpha & =\text { angle-of-attack, deg } \\ C_{N} & =\text { normal force coefficient } & \beta & =\text { sideslip angle, deg } \\ C_{m} & =\text { pitching-moment coefficients } & \phi_{k} & =\text { phase angle, deg } \\ \bar{c} & =\text { mean aerodynamic chord, } \mathrm{ft} & \sigma & =\text { standard deviation } \\ R^{2} & =\text { coefficient of determination } & & \\ S & =\text { reference area, } \mathrm{ft}^{2} & \\ T & =\text { period, sec } & & \\ V & =\text { velocity, fps } & \end{array}$

\section{Introduction}

$\mathrm{T}$ he GL-10 prototype aircraft efficiently combines two challenging mission objectives: long endurance and Vertical Take-Off and Landing (VTOL) flight. At full scale the design exploits the advantages of a hybrid diesel electric drive train, namely, scalable Distributed Electric Propulsion (DEP). Exploiting this advantage allows an efficient airframe design with light weight propulsion providing sufficient power required for vertical takeoff as well as efficiently providing significantly lower power required in forward flight for 24 hour endurance. This increase in efficiency causes a proportional increase in control system and aerodynamic modeling complexity. Propulsion and aerodynamic interactions add to the aerodynamic modeling and control design complexity. Additionally, the aircraft

\footnotetext{
${ }^{1}$ Research Engineer, Dynamic Systems and Control Branch, MS308.

${ }^{2}$ Senior Research Engineer, Dynamic Systems \& Control Branch, MS 308, Associate Fellow.

${ }^{3}$ Senior Research Engineer, Dynamic Systems \& Control Branch, MS 308, Member.

${ }^{4}$ Senior Research Engineer, Dynamic Systems \& Control Branch, MS 308, Associate Fellow.

${ }^{5}$ Senior Research Engineer, Dynamic Systems \& Control Branch, MS 308, Member.

${ }^{6}$ Senior Research Engineer, Flight Dynamics Branch, MS 308, Associate Fellow.

${ }^{7}$ Senior Research Engineer, Flight Dynamics Branch, MS 308.
} 
must traverse a difficult flight mode between hover and wing born forward flight, the transition flight mode. This distributed propulsion approach, though complex aerodynamically, offers advantages: excess power in transition, propulsive control power in all flight modes, beneficial downwash over the wing and tail during low speed transition providing some control surface control power, and downwash that creates a lower wing incidence angle during transition avoiding stall.

This project began with mission requirements and an extensive period of conceptual design during which many aircraft configurations were considered (see Ref. 1 to see a few of the additional concepts). Two concepts out of the mix were chosen to prototype. A fascinating concept, called the Dos Samara, is under development by Joby Aviation to meet the same mission requirements. The GL-10 concept is under development at NASA Langley. The GL-10 bears a strong resemblance, in terms of configuration, to the XC-142 and CL-84. Both aircraft are VTOL tiltwing transports developed around the 1960 s to 1970 s. ${ }^{2,15}$ The XC-142 is a tilt-wing with four propellers along the wing, an all moving horizontal stabilizer, and one tail propeller used for pitch control. As is often the case, most permutations of basic configurations were proven by predecessors. The GL-10 concept takes the well proven tiltwing aircraft configuration and combines distributed electric propulsion in a concept that provides the new capability of extremely efficient aircraft operations fully exploiting salient features of both. So, the primary challenge for the GL-10 project is not proving the basic aircraft configuration feasibility, as tilt-wings do indeed fly, but developing the flight control and flight test feasibility given schedule, resource constraints, and additional complexity from concurrent development for portions of the conceptual design, fabrication, and control development. Five main technologies are leveraged:

1) Efficient tunnel testing using Design Of Experiments (DOE) technique targeting short test time using a low-cost model and facility

2) In house Simulink® toolbox developed to quickly create representative simulations that can be easily modified

3) L1 adaptive control structure

4) In-flight system identification for model refinement and validation in key areas of the flight envelope

5) The Avionics System for Remotely Operated Vehicles (ASROV): a capable modular avionics system with reconfigurable digital input output

The following describes the approach used in this on-going effort to design a flight control system for the GL-10. A concept overview is provided to explain the advantages of distributed electric propulsion and its application in this prototype. The aerodynamic model development approach overview examines use of a fully actuated wind tunnel model coupled to an automated wind tunnel data acquisition system. DOE techniques used are shown to provide an efficient experiment design for varying the large number of factors required to define a comprehensive static aerodynamic model. A representative simulation is discussed which required treatment of the multi-body interactions and constraint forces acting through actuation mechanisms. Models of propulsive forces and moments, vehicle aerodynamics, actuator dynamics for all 21

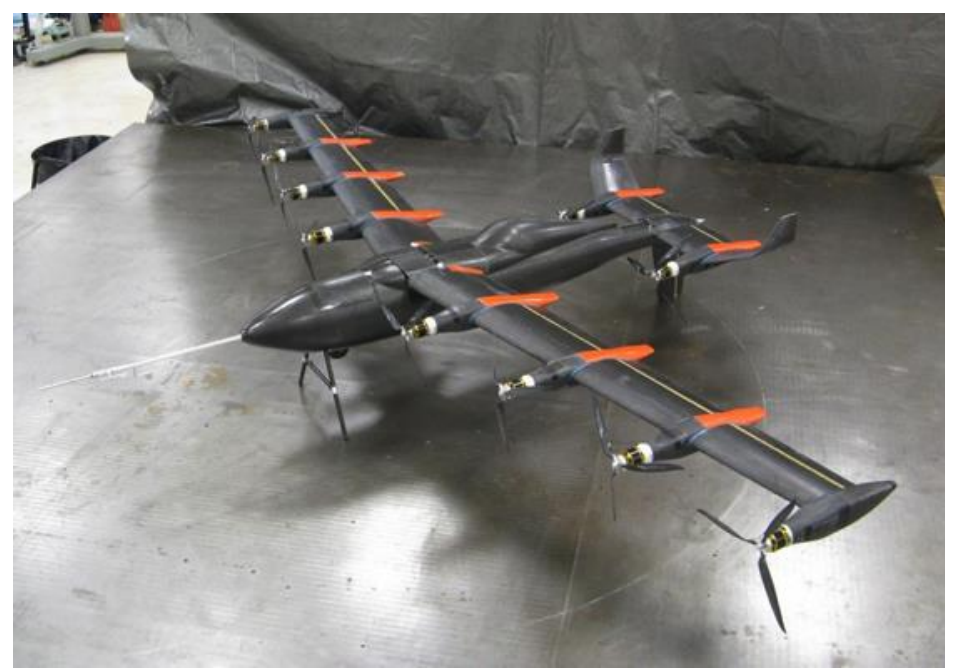

Figure 1. The GL-10 50\% scale all-electric prototype aircraft weighing approximately $60 \mathrm{lbs}$ with a $10.5 \mathrm{ft}$ wingspan, actuators, and multi-body dynamics for 13 bodies, are combined in software to produce a simulation. L1 adaptive control is to be used to increase robustness to uncertainty throughout the flight envelop. The approach to control algorithm implementation, avionics, and general flight testing operations is highlighted. An overview of the in-flight system identification techniques planned for validating wind tunnel aerodynamic models is presented. 

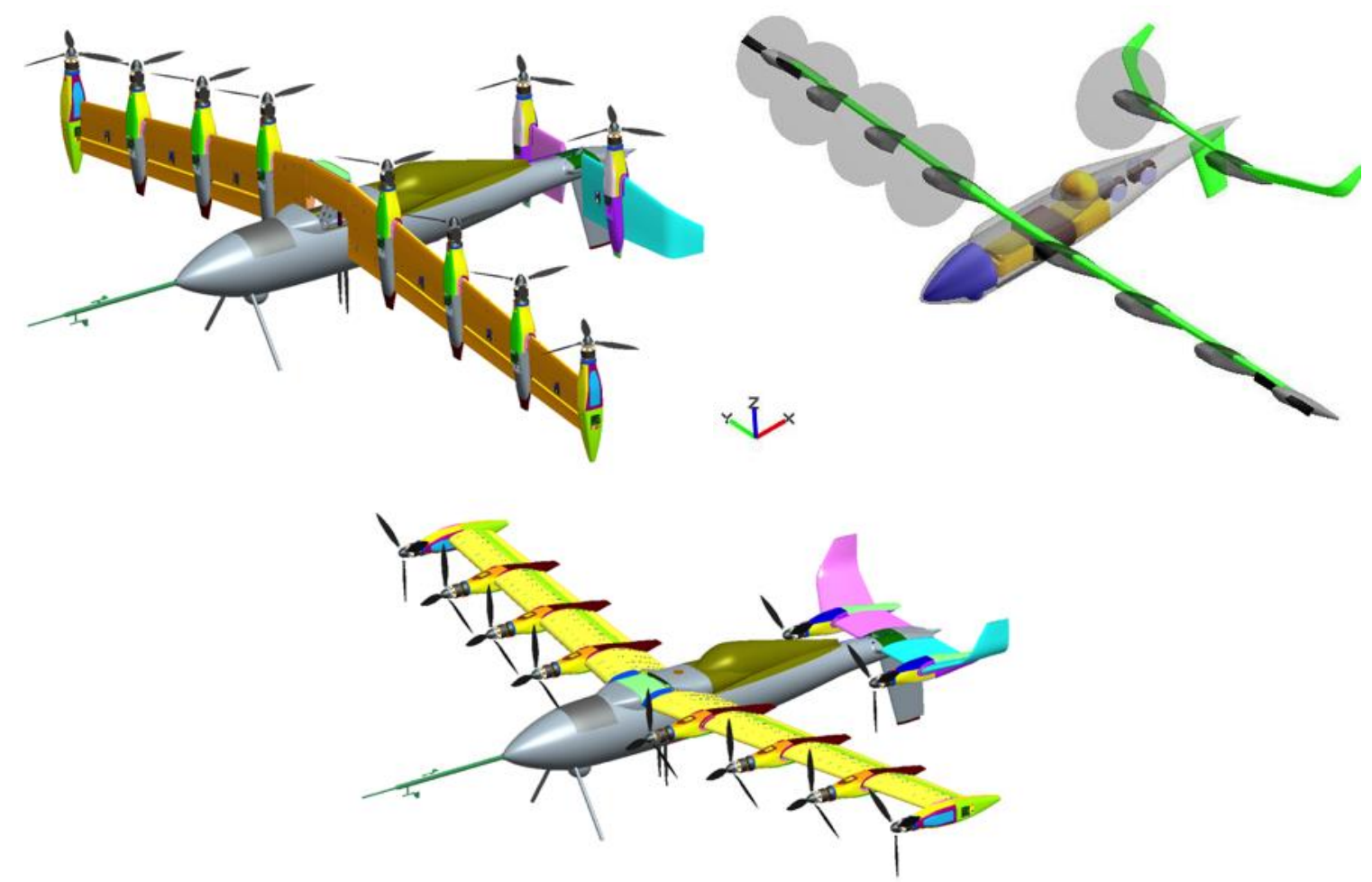

Figure 2. GL-10 CAD models showing different flight modes: hover, transition, and cruise.

\section{Distributed Electric Propulsion Conceptual Design Advantages}

The GL-10 is an experiment in combining Long Endurance and Vertical Take-Off and Landing (LEVTOL) operations using a series hybrid electric propulsion system and DEP. There are many advantages for using electric propulsion on aircraft: power to weight $(\mathrm{P} / \mathrm{W})$ ratios exceeding $5 \mathrm{HP} / \mathrm{lb}$, direct drive propulsion, compact form factor, high efficiency over the majority of motor speed range, ability to overpower motors over $2 \mathrm{x}$ the continuous power ratings for short bursts, and the most significant, electric motors are effectively scale-free meaning the power to weight ratios, efficiencies, etc. are the same independent of motor scale. This scale-free advantage of electric motors allows the designer new freedom in conceptual aircraft design; the propulsion system can be distributed all over the airframe. So, one can then "put propulsion where the drag is", reclaim drag losses, and take advantage of other useful synergies: no mechanical transmissions, structural mass reduction from mass distribution over wing span, redundancy without mass penalty, etc. This new design space encourages unconventional aircraft configurations meeting novel and challenging mission requirements that would be impossible, or minimally cumbersome and unreliable, to attempt using internal combustion or turbine engines. One such mission requirement is the combination of VTOL capability with long endurance. Electric propulsion provides synergy between these diametrically opposed mission requirements by usage of its scale free advantages and its overpower capability. This solves the longstanding problem of power matching the high power requirement of VTOL flight with the significantly smaller cruise power required for long endurance. ${ }^{2}$

\section{Project Objectives and Overview}

The final project objective is a fully autonomous system capable of vertical takeoff, GPS waypoint navigation, and vertical landing at an unimproved landing site. Towards this end, a significant intermediate milestone is a Radio Controlled (RC) flight test of an all-electric 50\% scale model of the GL-10 using an experienced RC pilot and active feedback stabilization. This flight is aimed at validation of the flight control concept for this aircraft and collection of performance data to validate predictions concerning aircraft range, endurance, etc. Several strategies can be engaged to meet these objectives. The project employed a hybrid of DOE tunnel testing mixed with model refinement using in-flight identification and control algorithm testing using lower cost, smaller scale aircraft. A build-up approach was selected for flight testing. Several aircraft scales with similar geometric configuration were 
fabricated to aid the pilot in training on an aircraft in type. Several aircraft were produced at approximately $15 \%$ scale solely for pilot training. Multiple 35\% scale aircraft called the Greased Lightning Almost Ready to Fly (GLARF) built from a modified almost-ready-to-fly kit were used for pilot training on an airframe closer in weight and performance to the half-scale prototype. The GLARF also uses avionics hardware and algorithms identical to the 50\% scale prototype. The GLARF serves as a work horse airframe that has little consequence for failure due to the airframes low cost. This allows the control designers to test a control algorithm structure early. This approach may be suitable for accomplishing a piloted demonstration, but a detailed simulation is necessary to develop a capable autopilot. A wind tunnel test was performed to develop an aerodynamic model suitable for control design and analysis. The aircraft equations of motion were developed to account for multi-body effects including the gyroscopic effect of each propeller and the reaction forces between bodies. The aerodynamic modeling was chosen by design to have greater confidence intervals in an effort to reduce test time and cost. Adaptive control algorithms robust to modeling uncertainty are planned for use to ensure the aircraft can be piloted through maneuvers for inflight system identification to develop a higher fidelity model. So, the overall approach for model development is to first collect the minimum amount of data required from ground/tunnel testing to create a representative simulation and design controllers with an emphasis on robustness. Next, several different scale aircraft are to be flown into uncertain or unexplored regions of the flight envelope and system identification experiments performed to refine the models. Finally, flight control algorithms are redeveloped to an acceptable level of performance to begin autopilot navigation development and demonstration.

\section{Aerodynamic Development}

Two requirements for GL-10 model development were to characterize aerodynamics in a relatively short period of time and at a relatively low cost. Considering this the project determined that somewhat greater uncertainty could be tolerated. The GL-10 all-electric prototype control system commands 21 control variables, ten of which are propulsion commands. Two of these control signals, wing tilt and tail tilt, significantly change the vehicles geometric configuration and move the overall center of gravity. The GL-10's flight envelope encompasses a large range of vehicle configurations, airspeeds, and flight attitudes. Including angle of attack and sideslip, 23 variables or factors required investigation in order to properly model this vehicle.

Characterizing GL-10 aerodynamics under these requirements presents a number of challenges to conventional methods of testing where one factor at a time (OFAT) is varied while holding all other variables constant. The first challenge is the long test time required due to the large number of factors involved. A test matrix to map 23 factors and their interactions would take a test duration the project found prohibitive. The second challenge is handling system errors. The conventional method assumes the test apparatus remain stable during the testing period, although this assumption is frequently checked. Corrections for temperature drift, zero drift in the measurement systems, etc. can be made, but this becomes more difficult for longer test runs. In the case of the GL-10 experiment collecting one block of data points typically took hours. Unless data for corrections is being collected over the course of a run, the errors due to the testing system become indistinguishable from precision errors and cannot be separated or quantified. A third challenge is that the conventional approach requires significant effort to detect response changes due to interactions between input variables which can significantly increase the size of the test matrix. Obtaining cross products (interactions) in the response polynomial models require input designs that produce responses with the proper information content to allow identification of these and other higher order terms.

The solution to these challenges was a 23 factor experiment designed using Design of Experiment (DOE) theory ${ }^{2}$ that produced extremely efficient, statistically rigorous experiment designs that minimized prediction error. DOE theory gives researchers a methodology for defining experiments that are very efficient in estimating the effects of input variables, their interactions, and sensitivities of the responses to these inputs. This is done in a statistically rigorous framework that allows optimizations of the designs to produce more robustness with respect to various types of error. In addition, the experimenter can a priori determine how much statistical power is available for the design and ensure that sufficient data are collected to achieve the desired statistical performance.

The testing was also facilitated by specific mechanical automation and software capabilities provided in Langley's 12-Foot Wind Tunnel discussed in Ref. 3. These capabilities allowed the basic principles of DOE to be incorporated into the test. Four principles in DOE theory have a direct bearing on the wind tunnel setup and operational requirements:

1. Orthogonal regressors - to improve estimation calculations

2. Replication - independent and repeated measurements.

3. Randomization - randomized input test matrix. 


\section{Blocking - technique to improve precision}

Effectively these requirements on the test facility are accommodated by three capabilities in the test facility: automation, speed, and accurate arbitrary motion. Automation addresses replication and randomization by providing the abilities to command tunnel state, model support system (sting position), and all model actuators from a predefined user test matrix, followed by recording of input commands, achieved set points, and aerodynamic responses. Achieving set points accurately is key to maintaining orthogonal regressors and good replication. Ideally this information is collected as fast and accurately as possible and then transmitted back in the exact sequence tested to the investigators for analysis. Rapid data acquisition is a key factor that allows a very large number of test points to be collected in a short time. Precision is also helped by incorporating blocking into the experiment design to remove the effects of known sources of error.

\section{A. GL-10 Experiment Design}

GL-10 model characterization was accomplished using three distinctly different types of experiments: exploratory, DOE, and engine-only tests without the airframe present. A limited set of exploratory tests were followed by four main DOE tests reflecting the four modes of GL-10 operation: (1) Cruise, (2) Loiter, (3) Transition, and (4) Hover. After these tests a series of tests for one engine and propeller without the airframe were performed over a large range of incidence angles and power settings.

Exploratory experiments were performed using both DOE and conventional test methods to observe where nonlinear aerodynamic behaviors occur and resolve several key issues. Key issues addressed in advance of the main DOE test were:

1. Determine minimum duration of data samples required.

2. Determine presence and severity of aerodynamic hysteresis effects.

3. Determine degree and location of aerodynamic nonlinearities.

4. Determine if vortilons were required.

5. Obtain representative samples of OFAT data for comparison with DOE results.

These issues were quickly resolved by a series of short tests. Given the large test matrix for the main test it was important to determine the minimum sample time that would adequately estimate mean aerodynamic forces and moments in any region of the flight envelope while maintaining statistical accuracy. This test was performed using a DOE test matrix and results are shown in Section B. Conventional static tests over angle of attack and sideslip highlighted the presence and severity of any static aerodynamic hysteresis effects. These data lead to a decision that static hysteresis effects, although present in some regions, did not need to be included in the model. Consequently the experiment was not designed to incorporate those characteristics and the resulting slightly larger measurement errors were acceptable. Conventional tests were also used to determine the degree and location of aerodynamic nonlinearities. These tests were necessary to define the appropriate ranges for major factors in the experiment such as angle of attack, sideslip, and propeller speed. Another test determined if vortilons were required to improve flight performance in high lift conditions. These were found to have limited effect and were eliminated from the test matrix. Limited additional OFAT data were obtained to provide representative samples of basic aerodynamics and control effectiveness for comparison with DOE results.

The main design objective for the DOE test was aerodynamic characterization of the GL-10 configuration. The aerodynamic model was required to support simulation of all flight modes and flight control law design. The test design had to account for a complex aircraft with 9 surfaces, 10 engines, wing tilt, tail tilt, angle of attack and sideslip. Due to the large number of factors a very efficient and effective test methodology was required.

Some key contributions, such as in Refs. 4-6, have been made in the application of DOE for aerodynamic modeling. Reference 5 describes application of DOE to the Boeing Blended Wing Body configuration. In this case the numerous surfaces were ganged reducing the total number of factors to 8 . An efficient fractional factorial design was created to capture main effects, two factor and three-factor interactions. In Ref. 6 a novel design, describes as a nested-face-centered design (FCD), was created for the X-31 aircraft with 5 factors. This design incorporated the advantages of a fractional FCD and ensured 5 equal levels for control surface deflections that cover the full range of motion. Given the complex nature of GL-10 with 23 factors, the current study extended the previous work by blending nested FCD designs with IV-optimal designs. The optimal designs minimize prediction error.

\section{B. Sample GL-10 Modeling Results}

In Cruise mode GL-10 operates as a conventional aircraft. Wing tilt is not used, tail tilt is limited to $\pm 5^{\circ}$, and only two outboard engines provide propulsion. This leaves only 14 factors for this mode to be tested. Loiter mode is configured the same as the Cruise mode but in this mode GL-10 can operate in the region of stall angle of attack. In 
transition mode all 23 factors are used in the design and full motion is available to wing and tail-tilt angles. In hover mode wing-tilt and tail-tilt are locked at $90^{\circ}$, reducing the number of factors to 19 . For the transition mode two separate blocks of data were collected. A low transition mode (TransLo) that covered wing-tilt from $0^{\circ}$ to $45^{\circ}$ and a high transition mode (TransHi) with wing-tilt ranging from $45^{\circ}$ to $90^{\circ}$.

As an example of the DOE designs for GL-10, design test points are shown for the TransLo mode in Fig. 3. The figure shows a nested FCD blended with optimized test points that minimize prediction error. Angle of attack and sideslip are the two factors selected for this plot but this is a representative set of test points for all the modes and factors. The limited ranges for angle of attack $\left(0^{\circ}\right.$ to $\left.15^{\circ}\right)$ and sideslip $\left(-5^{\circ}\right.$ to $\left.5^{\circ}\right)$ reflect the expected flight envelope in this mode. The four colors black, red, green, and blue, correspond to the four design test blocks 1 to 4 , respectively. Blocks 1 and 2 define a nested FCD and blocks 3 and 4 are the optimized test points that minimize prediction error. Block 1 (black) is the outer FCD (8 points) and block 2 (red) is the inner FCD. Optimal points in blocks 3 and 4 can replace original FCD points (outer or inner/nested FCD) if the optimizer determines them to be

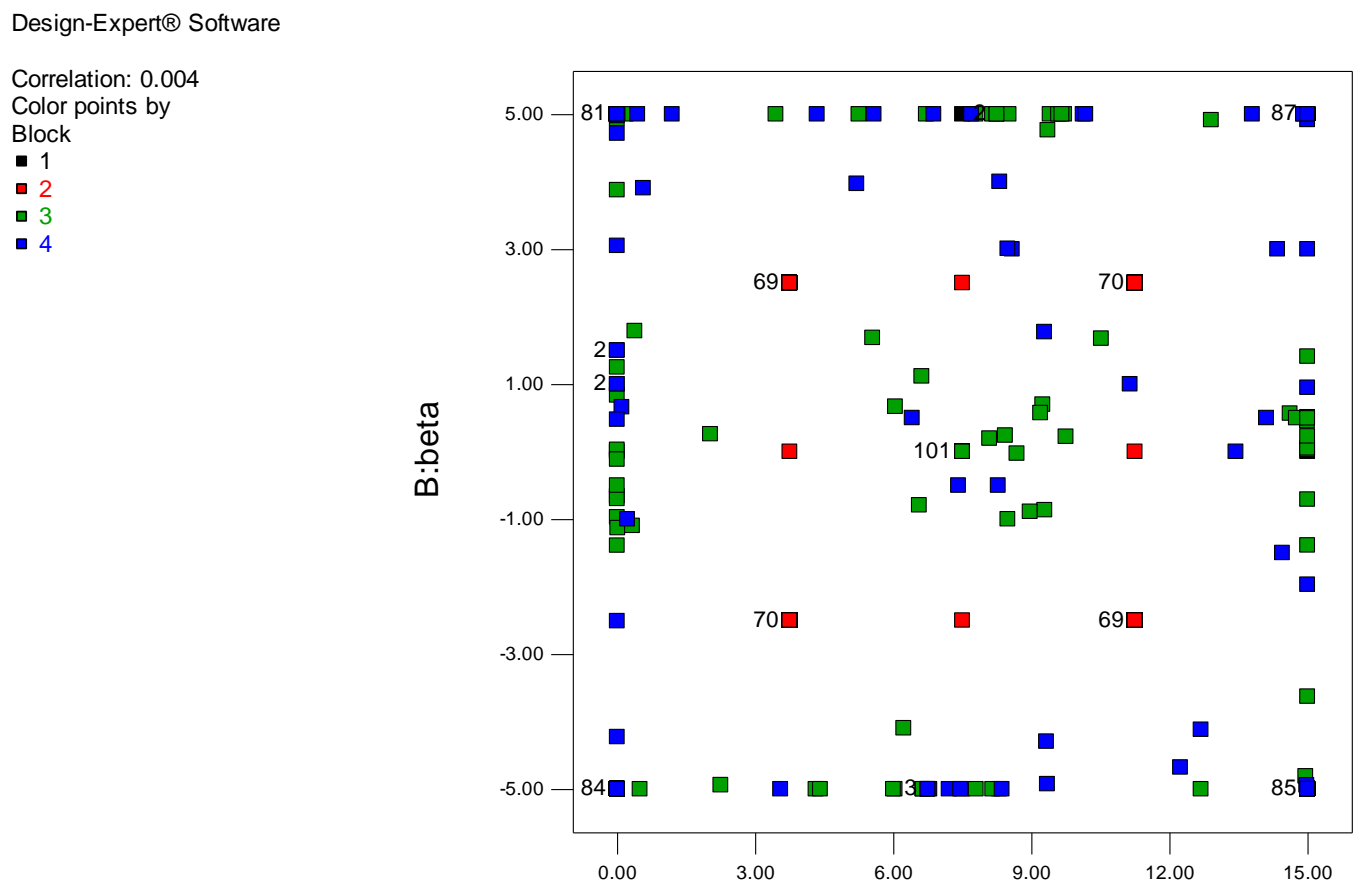

A:aoa

Figure 3. Nested FCD blended with optimized test points for TransLo mode.

required test points. Either way the underlying nested FCD is always tested. Typically corners and centers are very key test points, hence the nested FCD is effective even by itself.

For the TransLo mode, modeling work found that a predominately quadratic model for normal force with limited cubic terms associated with angle of attack and wing-tilt is adequate. This model covers all main effects, 2 -factor interactions, and some cubic behavior of normal force. A surface plot showing the estimated normal force model as a function of angle of attack and wing-tilt (Wtilt) is shown in Fig. 4. Measured values are presented on the chart as red dots if they are above the predicted surface and pink dots if they are below. Limited measured values are shown for clarity. Five measured values are shown at the center of the range for each independent factor. The remaining factors were set to their mid-range values to create this surface. The factor values are shown in the legend. A similar graphic is presented in Fig. 5 for the high range transition mode (TransHi). In this case a relatively flat surface is presented for normal force against the same two factors. However, for TransHi the analysis found that limited cubic terms were still statistically significant and required for the polynomial model. 
Design-Expert® Software

Factor Coding: Actual

$\mathrm{CN}$

- Design points above predicted value

- Design polints below predicted vahte

4.22759

$-0.523311$

$\mathrm{X} 1=\mathrm{A}:$ aoa

$\mathrm{X} 2=\mathrm{L}:$ Wtilt

Actual Factors

$\mathrm{B}:$ beta $=0.00$

C: LWS1 $=-7.50$

D: $L W S 2=-7.50$

E: LWS3 $=-7.50$

$\mathrm{F}: \mathrm{RWS4}=-7.50$

G: RWS5 $=-7.50$

$\mathrm{H}: \mathrm{RWS6}=-7.50$

$\mathrm{J}: \mathrm{LTS} 1=-7.50$

$\mathrm{K}: \mathrm{RTS} 2=-7.50$

M: Ttilt $=21.00$

$\mathrm{N}: \mathrm{Rud}=0.00$

O: LWE1 $=8000.00$

P: LWE2 $=8000.00$

$\mathrm{Q}:$ LWE3 $=8000.00$

R: LWE4 $=8000.00$

S: RWE5 $=8000.00$

T: RWE6 $=8000.00$

$\mathrm{U}: \mathrm{RWE7}=8000.00$

$\mathrm{V}:$ RWE8 $=8000.00$

W: LTE1 $=8000.00$

$\mathrm{X}:$ RTE2 $=8000.00$

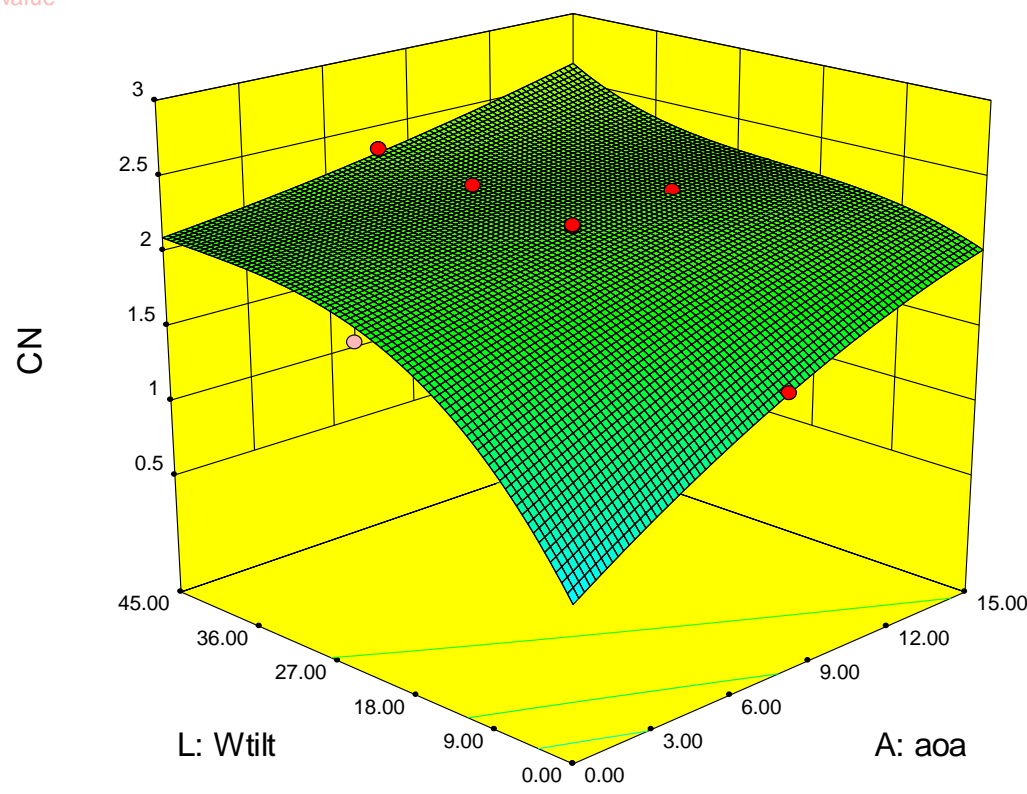

Figure 4. Normal force as a function of angle of attack and wing-tilt in TransLo Mode.

Design-Expert@ Software

Factor Coding: Actual

$\mathrm{CN}$

- Design points above predicted value

- Design points below predicted value

4.15069

0.612705

$\mathrm{X} 1=\mathrm{A}: \mathrm{aoa}$

$\mathrm{X} 2=\mathrm{L}:$ Wtilt

Actual Factors

B: beta $=0.00$

C: $L W S 1=-7.50$

D: LWS2 $=-7.50$

$E:$ LWS3 $=-7.50$

$\mathrm{F}: \mathrm{RWS4}=-7.50$

G: RWS5 $=-7.50$

$\mathrm{H}:$ RWS6 $=-7.50$

$\mathrm{J}: \mathrm{LTS} 1=-7.50$

$\mathrm{K}: \mathrm{RTS} 2=-7.50$

M: Ttilt $=71.50$

$\mathrm{N}: \mathrm{Rud}=0.00$

O: LWE1 $=8000.00$

$P:$ LWE2 $=8000.00$

Q: LWE3 $=8000.00$

R: LWE4 $=8000.00$

S: RWE5 $=8000.00$

T: RWE6 $=8000.00$

$\mathrm{U}: \mathrm{RWE7}=8000.00$

V: RWE8 $=8000.00$

W: LTE1 $=8000.00$

$\mathrm{X}: \mathrm{RTE2}=8000.00$

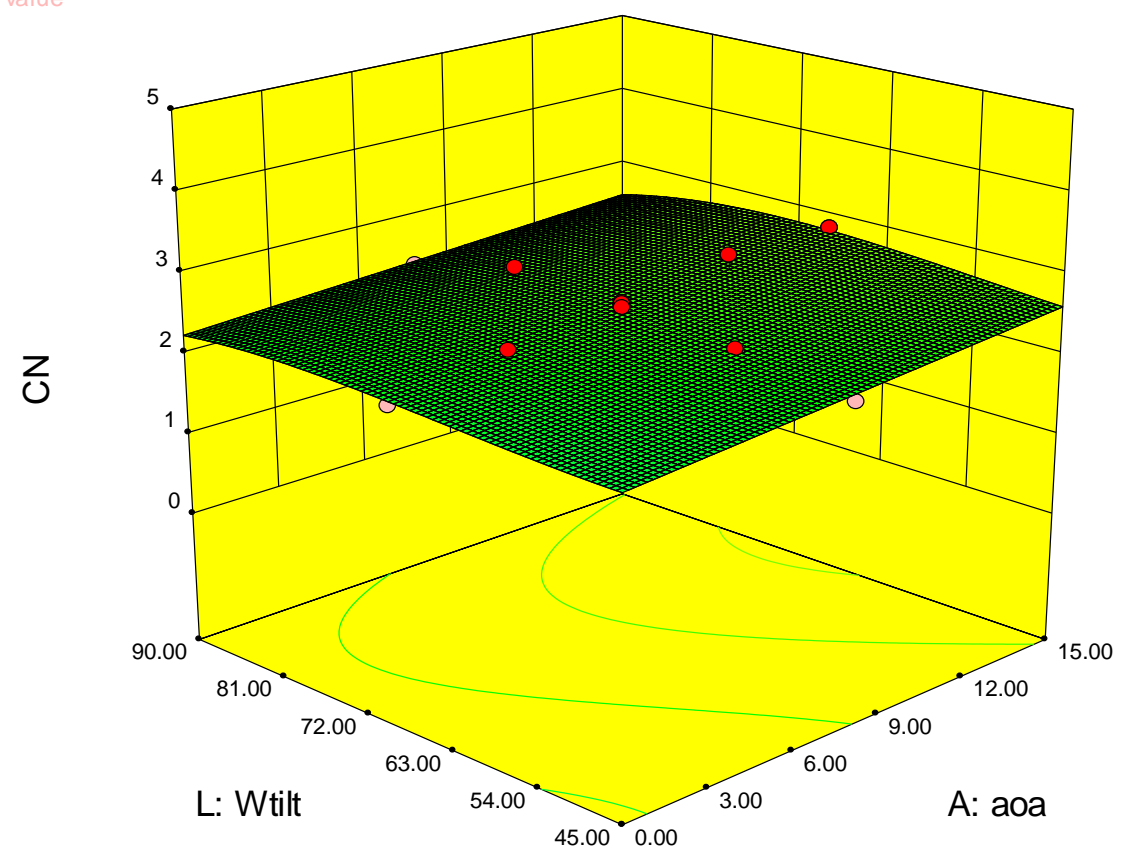

Figure 5. Normal force as a function of angle of attack and wing-tilt in TransHi Mode.

American Institute of Aeronautics and Astronautics 


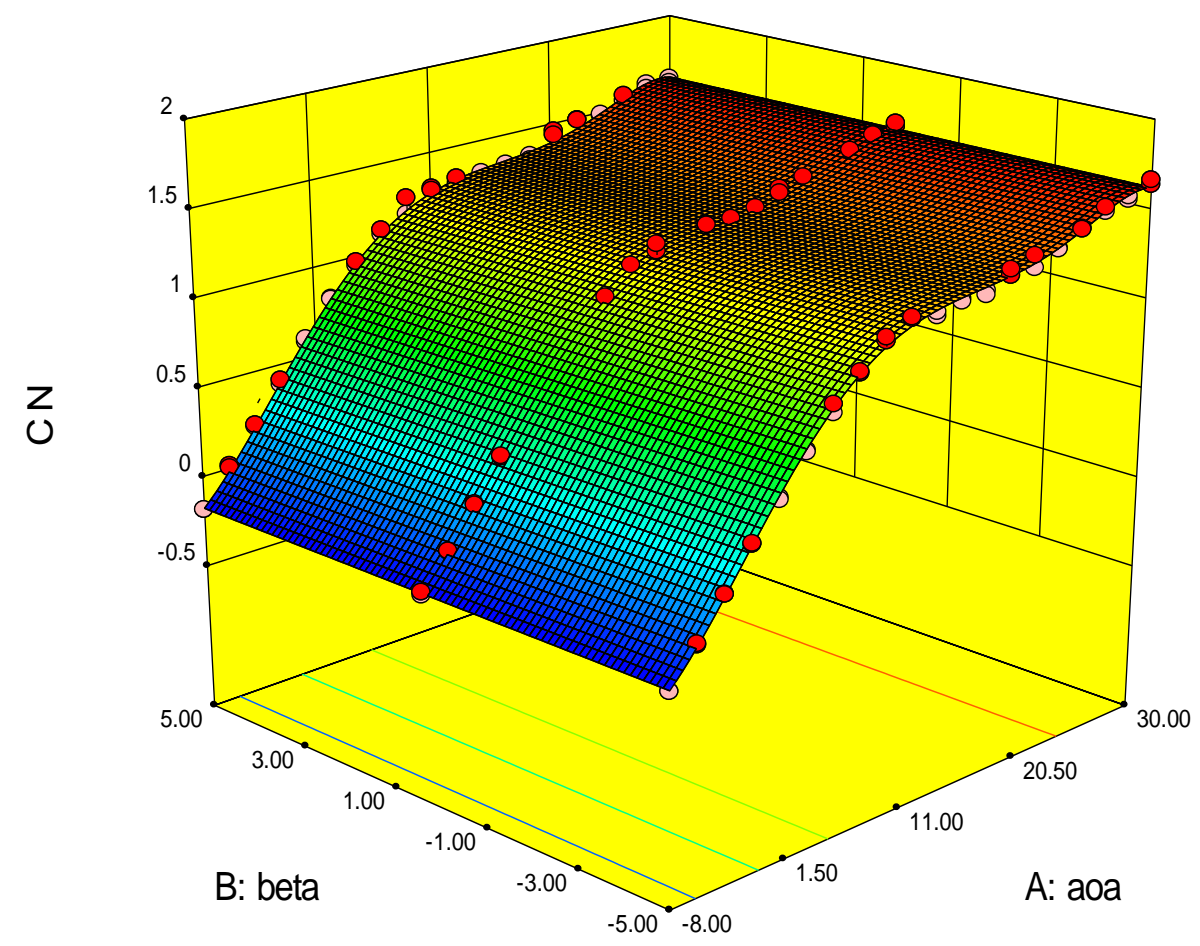

Figure 6. Alpha sweeps of Gl-10 with propellers removed shown here to highlight the smooth GL-10 normal force curve through stall

\section{Interesting DEP Aerodynamic Feature}

Analysis for the GL-10 wind tunnel test is on-going, though one extremely interesting characteristic of the aircraft bears mention. The aircraft's lift coefficient remains relatively flat beyond stall at 10 degrees angle of attack. The results seen in Fig. 6 and 7, show the aircraft normal force results and lift coefficient curves from some traditional alpha sweeps with propellers removed from the aircraft. These sweeps were run as checks for hysteresis and for future analysis to compare traditional tunnel testing methods with the DOE techniques. No comparisons between the methods are implied here. The shape of the normal force model here with propellers removed corroborate results seen in the normal force plots in Fig. 4 above, which include powered propulsors and wing tilt. The researchers hypothesize that the motor pylons stationed on the wing act as huge vortilons, shedding powerful vortices over the upper wing surface during high angles of attack. The other aerodynamic parameters are under study. It has not been concluded how well the rest of the aerodynamics behave during high-alpha flight, though the benign effects of stall on lift are certainly an advantage for a transitioning VTOL aircraft.
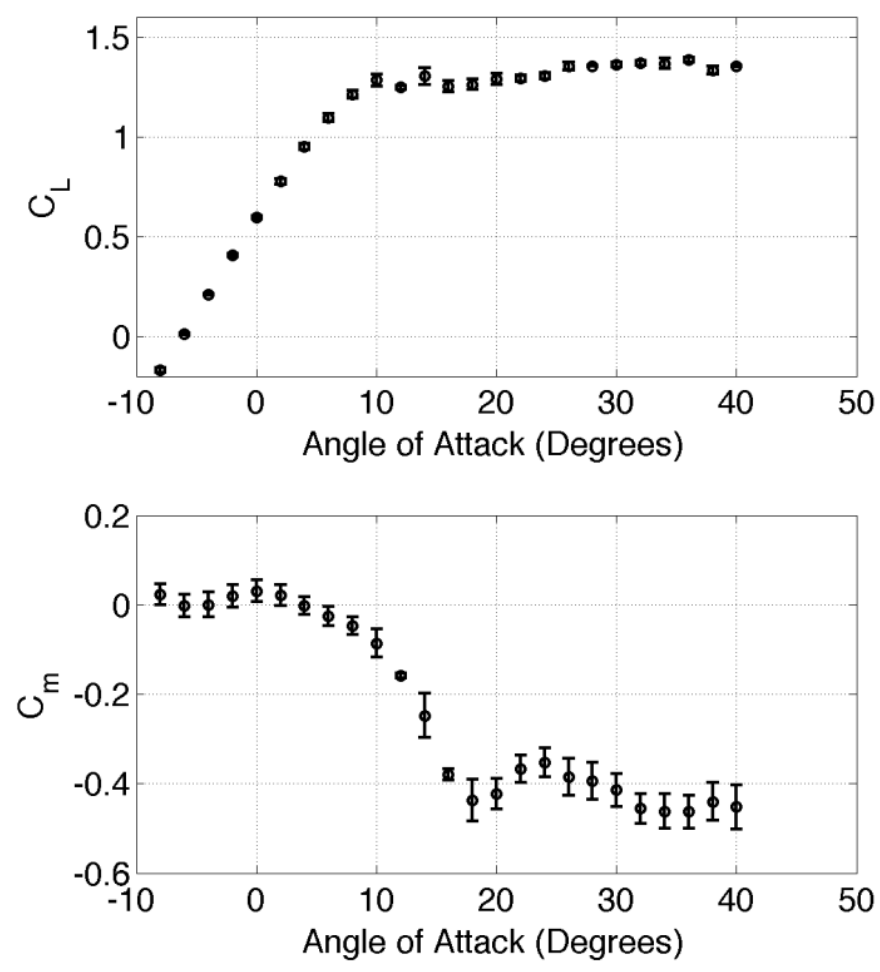

Figure 7. GL-10 Lift, drag, and pitching moment coefficients function of angle of attack with propellers removed at zero sideslip angle. GL-10 has moderately flat lift curve beyond stall. 


\section{Propeller Testing}

In addition to the GL-10 tunnel testing, individual propellers were tested over large angles of attack. Three different propellers were tested: a 9.0 inch diameter propeller with a 4.5 inch blade pitch (9" $\mathrm{x} 4.5$ ") was used on the wind tunnel model, a 11"x5.5" propeller used on the GLARF 35\% scale airplane, and the 16"x8" propeller used on the $50 \%$ scale GL-10. Both the smaller diameter propellers are two bladed rigid propellers. The 16 " $x 8$ " propeller is a 3 bladed hinged (folding) propeller. The propellers were run from 0 to 110 degrees angle of attack at several different airspeeds. At each data station, a range of propeller speeds were tested to encompass the advance ratios expected for the respective vehicles flight conditions. As expected, the tests results show a significant difference between the hinged and rigid propellers especially in the propeller normal force and pitching moment with the rigid propellers being far better behaved. The effects of both a rigid and hinged propeller were studied in flight tests using a $1 / 4$ scale VZ-2 by NASA in 1962, among others. It was determined the effects of the hinged propeller were certainly destabilizing but were manageable by the pilots. ${ }^{14}$ The approach chosen for the GL-10 project is to begin initial simulation studies with well behaved rigid propeller models then progress to the hinged propeller data when suitable experience is acquired. A propeller model was created for the flight vehicle using the thrust coefficient verses advance ratio (related to the ratio of free-stream velocity to tip speed) associated with the wind tunnel model's propeller. The thrust coefficient, power coefficient, and normal force versus advanced ratio were duplicated for the larger propeller and implemented in simulation. The hinged propeller data was used to create a simulation model as well to interchange with the rigid propeller model when more realism is desired in the design and testing process.

For a more complete compendium of the test techniques and automation features employed for the GL-10 wind tunnel experiment, see the companion paper to this publication in Ref. 3. This paper discusses in detail the tunnel automation, experiment groundwork, tunnel model fabrication, and practical aspects of testing the GL-10 in the NASA Langley 12-Foot Low Speed Wind Tunnel.

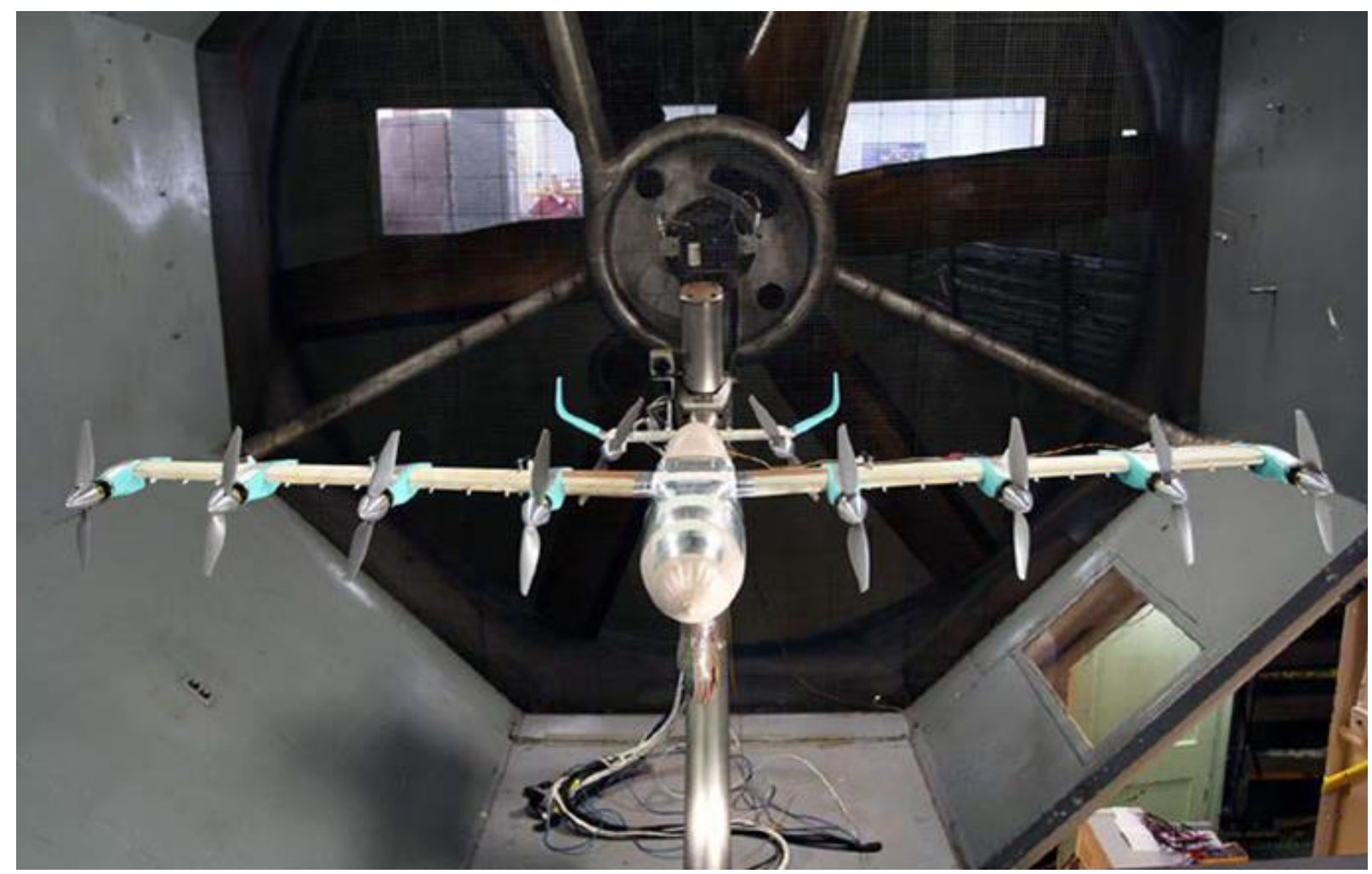

Figure 8. GL-10 28.125\% scale tunnel model in NASA Langley's 12-foot Low Speed Wind Tunnel

\section{Multi-body Equations of Motion, Actuator Modeling, and Simulation}

A multi-body derivation of the equations of motion was required for this vehicle for two reasons. Modelling propeller gyroscopic effects is one concern: capturing the effects of articulating lifting surfaces is another. First, depending on the magnitude of the propeller inertia, the gyroscopic moments created by ten spinning props are 
potentially non-trivial during transition to and from hover. Though the propeller speeds nominally cancel gyroscopic effects felt by the body, the propeller speeds may not always be varied differentially and may have very disparate speeds between rotors during demanding maneuvers. Second, if the relative mass properties of the articulating pieces are small compared to the overall vehicle's mass properties, the inertial loads induced by articulation are often calculated as if the vehicle is fixed in a test stand and added with the external forces and moments on the vehicle in a standard set rigid-body equations of motion. Moreover, moments due to the gyroscopic effects of propellers and rotors are also added as external moments to the standard set. For GL-10, this practice was not followed and equations of motion were developed for each body with the required moments for articulation supplied by actuator models. This included equations for the propeller and rotor subsystem, with reactions at the bearing and the electromagnetic motor housing transmitting the proper forces and moments to the lifting surface assembly. Both the actuator and electric motor models contained controls to reject disturbances due to changing flight conditions and maneuvering to respectively maintain the desired surface tilt and propeller RPM. A simple root-locus procedure provided the actuator and electric motor parameters necessary for modeling the appropriate dominant second-order responses.

Due to the preponderance of propellers and lifting surfaces on GL-10, the simulation utilized a Simulink® block set from the Space Transportation Analysis and Research Simulation (STARS) developed at NASA Langley Research Center. Although originally developed for launch vehicles, it has been used in launch abort systems and aircraft flying over rotating, oblate planetary bodies. One feature of STARS particularly useful to the GL-10 application is the block sets that perform operations over multiple input signals (sets of vectors, quaternions, $3 \times 3$ matrices, etc.). These operations include $3 \times 3$ matrix inversions and multiplications, cross products, quaternion operations, etc. This feature enabled fewer blocks in the simulation build up to realize the force and moment effects of two articulated lifting surfaces and ten propellers. As an example, the force and moment produced by the propeller is determined by the magnitude and orientation of the free stream velocity observed at the propeller hub, and is different for each propeller as the vehicle is maneuvering. The calculations and table-lookup required are the same for each propeller but each will have a different result that can be easily accommodated together with the block sets in STARS.

The DOE-based aerodynamic propulsive model posed two primary challenges: 1) a non-symmetrical regression fit of the wind tunnel data and 2) only one free-stream velocity tested for cruise, loiter, and transition. It was desired to have a simpler symmetric aerodynamic model for initial simulation and control development. A constrained regression fit enforcing symmetry was made based on the delivered models. In addition, a multiplication factor was applied to purely longitudinal regressors appearing in the lateral-directional coefficients, providing the user the ability to delete them from the response. The resulting model has the option of "turning on" the non-symmetric aerodynamics for controller checks but enables simpler symmetric aerodynamics for development purposes. The second problem arose because the propeller's forces and moments are dependent on free-stream velocity using a different set of non-dimensionalizing factors from the standard aerodynamic forces and moments. The problem is one of choosing the appropriate RPM for the wind tunnel model's propellers at one free-stream velocity to correspond to the flight vehicle propeller RPM at varying free-stream velocities. The solution was a RPM mapping that matched the thrust coefficient between the two vehicle's propellers. With this mapping, a suitable subtraction of purely propulsive effects could be performed on a dimensionalized version of aero/propulsion model at wind tunnel conditions and re-nondimensionalized to obtain the desired aerodynamic coefficients.

The resulting simulation captures the main aerodynamic, propulsive, and inertial effects of the GL-10 in all phases of flight. Features of the simulation include 1) a first-principle approach to addressing the multi-body problem, 2) a propulsion model that produces propeller axial and normal forces, and as stated, features a controller that maintains desired rpm for various flight conditions, and 3) an innovative DOE-derived aerodynamic model that required the extraction of the exclusive propulsive forces and moments from the wind tunnel model. The simulation also includes a trimming algorithm that works in all phases of flight (cruise, loiter, transition, and hover), and a linearization algorithm that translates quaternion-based models to Euler-based models with no change to the frequency response, particularly useful for control law development.

\section{Control Algorithm Approach Motivations}

The project deliberately made several choices early on to reduce cost and duration of flight control system development. One such decision was to use Commercial Off-The-Shelf (COTS) hobby grade hardware for the propulsion and actuation system on the aircraft. Though most of this hardware is reliable in its intended application, on GL-10 it is often applied slightly outside of its design parameters. Consequently, eventual actuator or propulsor failures during some portion of the GL-10 flight testing were expected. DEP aircraft tend to naturally have actuator 
redundancy. This feature makes the GL-10 concept configuration potentially robust to these types of failures. Hence, a control system architecture that can intelligently take advantage of the redundancy is desired. Additionally, the project chose to minimize cost and duration of wind tunnel testing for aerodynamic modeling at the expense of accepting greater uncertainty in the vehicle aerodynamics. Also, the aircraft is to be piloted which imposes additional requirements for enforcing appropriate handling qualities. Thus, at a minimum a suitable control system architecture must meet specific performance metrics while being robust to aerodynamic uncertainty (especially during transition), and actuator or propulsor failure. Based on its analytic performance predictability and flight test performance dealing with rapidly changing uncertain dynamics, ${ }^{7}$ L1 robust adaptive control architecture with control allocation capable of smoothly blending propulsive and surface control effectors ${ }^{8}$ has been chosen for the GL-10.

The number of potential control effectors, propulsive and aerodynamic, provides a degree of robustness but also presents an allocation problem. The control allocation problem for GL-10 is most challenging through transition. Unlike the XC-142 and other tilt-wing aircraft of the era, the GL-10 does not have a large vertical tail area, large rudder area, or dedicated pitch control propeller. This is an intentional design choice to increase endurance with the consequence of not having a large decoupled source of control power for directional or pitch control through transition. Thus, through the transition corridor the majority of directional control power must be derived from the wing propulsors and ailerons in the propeller slipstream. While the wing is at a large tilt angle, e.g. 45 degrees, ailerons produce strong coupled torques about vehicle body roll and yaw axes as do the wing propulsors. The CL-84 had a similar allocation problem (with significantly fewer actuators). A mixing strategy was employed ${ }^{15}$ that faded the ailerons from yaw control to roll control as the wing tilted from 90 degrees to 0 degrees. Similarly, a fade of differential thrust was used on the CL-84 to control roll in hover and yaw in forward flight. A similar strategy will be used for initial simulation of the GL-10, but the GL-10 has several additional options for allocation that its predecessors could not take advantage of that will be explored. An example of this is the common method used on quad-rotors for yaw control where differential thrust is commanded about all rotors to provided steady state yaw torques. A simple attempt to completely decouple control surface and propulsive torque production into pure body components is likely to quickly result in control surface saturation on GL-10 and not leave sufficient control authority for disturbance rejection and uncertainty compensation. To maximize decoupling while maintaining sufficient maneuver authority, it is likely the allocation will be chosen such that decoupling is about some axes other than the vehicle body axes such as wind axes, or most likely an axes system fixed to the wing. This choice strongly depends on the reference transition trajectory to be flown as significantly different wing incidence angles can be experienced depending on the trajectory chosen. Another complicating factor is the wing tilt actuation speed requires approximately 15-20 seconds for the full transition of 90 degrees. Considering the actuator speed limitations and the strong control torque coupling, the reference trajectory is a crucial component for robust transition performance. For instance, a maneuver may be performed in hover where the vehicle is trimmed to a fuselage angle of approximately 60 degrees angle of attack before losing pitch control power margin in hover. From this attitude the fuselage incidence can then tilt to adjust relative wing incidence and the trajectory could follow a level, descending or ascending flight path to forward flight. This eliminates much of the time required to linger in the transition region while waiting for the tilt actuation. Alternatively, the vehicle could perform a level fuselage, constant altitude trajectory while waiting for tilt actuation. There are many options. The choice of trajectory determines whether the aircraft is flying through a propulsion dominated or an aerodynamically dominated environment and as such likely results in a large difference in control power margin and consequently transition robustness. The project intends to perform a trajectory optimization study to find trajectories that maximize available control power throughout the transition from hover to level flight and vice versa while minimizing the change in altitude.

\section{Model Refinement Using System Identification}

Once a pilotable stabilized aircraft is available, system identification experiments are designed to further refine the aerodynamic model fidelity. System identification is the inverse problem of determining a useful and sufficiently accurate mathematical model of a system from observed measurements of inputs and outputs. In the present context, the model is the set of non-dimensional aerodynamic coefficients, the inputs are the control surface deflections and motor commands, and the outputs are the responses of the GL-10 aircraft. Although the aerodynamic coefficients were measured during the static wind tunnel tests, significantly different values are often extracted from measured flight test data. This is in part due to wind tunnel issues such as blockage and wall effects, but also because the aircraft responds in a free and coupled motion during flight. Identifying aerodynamic coefficients from flight test data can update the aerodynamic model for more accurate simulation, performance analysis, and control design. 
Flight tests for system identification are planned for the hover and slow forward flight modes of operation. Orthogonal phaseoptimized multisines will be used to excite dynamic responses for system identification analysis. ${ }^{9,10}$ These inputs have led to the successful modeling of numerous flight test vehicles, in both typical and unusual flight conditions. These inputs are well suited for the GL-10 flight tests because they efficiently excite all inputs simultaneously in an uncorrelated manner, resulting in relatively short maneuver lengths with high information content. Discrete frequencies are excited only within a specified band of interest to target specific responses. Additionally, peak amplitudes are minimized to keep the aircraft near the flight condition, which is good for perturbation modeling about reference flight conditions. These inputs have the form:

$u_{i}=\sum_{k \in K} a_{k} \sin \left(\frac{2 \pi k}{T} t+\phi_{k}\right)$

An example design for three inputs is shown in Fig. 9. Inputs such as these will be designed and superimposed onto trim inputs for the system identification flight tests.

A real-time frequency response estimation technique will be used during the flight test. ${ }^{11}$ This method recursively transforms input/output data into the frequency domain to estimate frequency response data and uncertainties. This method is suitable for the GL-10 because it does not require a model structure, and can be applied to many input/output channels because of its computational simplicity. These real-time modeling results, which update during the flight test, will be used to diagnose instrumentation problems, assess modeling quality, and estimate control effectiveness and stability margins.

After the flight test, standard signal processing and data compatibility techniques, as discussed in Ref. 9, will be used to prepare the data for additional system identification analysis. Multivariable orthogonal function modeling, ${ }^{12}$ will be used to determine the functional dependence of the aerodynamic model structure. This method uses a statistical metric to automatically select the minimal number of modeling terms from a provided set of explanatory variables that best model the data. Afterwards, equationerror and output-error parameter estimation methods ${ }^{9}$ will be employed to estimate coefficients in that model structure that determine the best parameters that match the model outputs to the measured outputs. Additional flight data will then be used to validate the prediction accuracy of the identified model.

\section{Control Implementation and Avionics}

An aircraft such as the GL-10 is rich with avionic complexity. The aircraft in its early ideation had well over 30 actuators including variable pitch propellers, conventional control surfaces, retractable
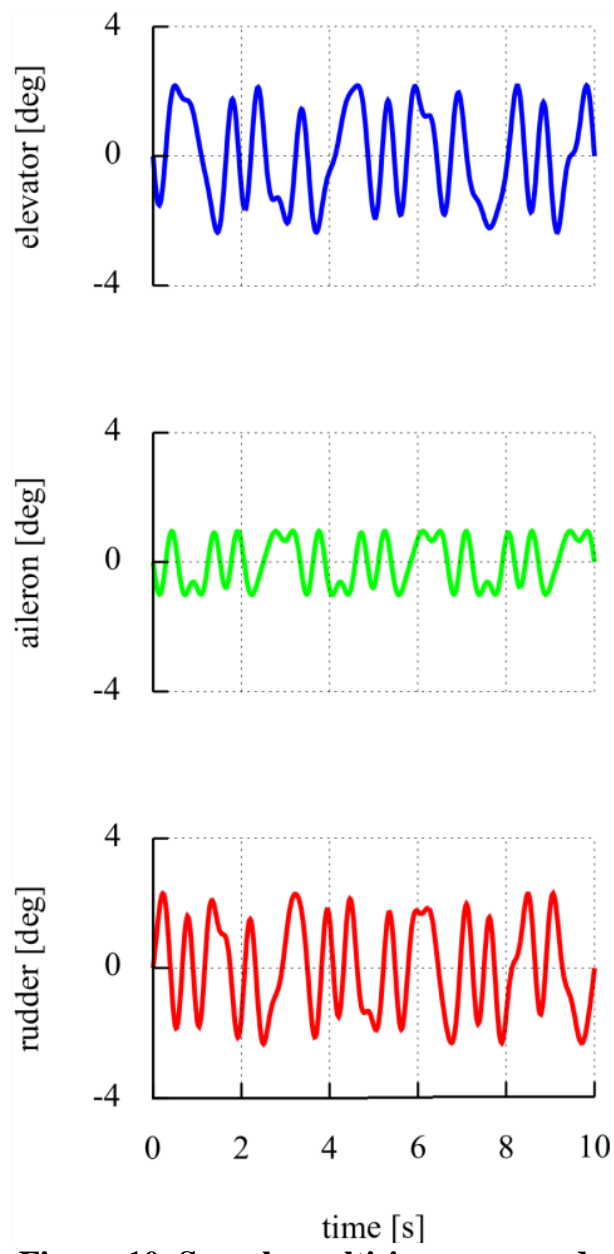

Figure 10. Sample multisine commands to send to three sets of actuators. 
landing gear, wing and tail tilting mechanisms etc. After simplification, for easier prototyping and testing, 21 actuators are used on the GL-10 50\% scale prototype. This number of actuators alone ruled out many low cost avionics packages. The NASA Langley AirSTAR project developed, under SBIR contract with Coherent Technical Services, Inc., a capable avionics system called the Avionics System for Remotely Operated Vehicles (ASROV). The ASROV system is comprised of a stack of PCI/104 form factor computer boards. It has a dual-processor architecture allowing research control algorithms to be run on the secondary processor (ResFCS). Safety logic and baseline control algorithms can be run on the primary processor (PriFCS). PriFCS is also equipped with a Field Programmable Gate Array (FPGA) allowing custom digital logic to be created and reconfigured as required. The system can be run with one or two 32 differential channel analog to digital converter cards. Depending on the stack configuration and enclosures used the system can weigh between 1-6 lbs. All AirSTAR avionics systems, ASROV included, are configured to accept auto-coded MATLAB/Simulink control diagrams. This allows control design engineers to rapidly implement control algorithm changes, on the order of a few minutes. This feature plus complete access to the system source code provide significant advantage for the GL-10 compared to many alternative small aircraft avionics platforms.

Future goals of the project include extremely low-cost airframes to allow low-risk flight testing of experimental control algorithms. An effort was made toward reduction of instrumentation cost without penalty in function or data quality. An example of this effort is a digital air-data probe with sufficient resolution for system identification that was designed, fabricated and interfaced with ASROV. It was desired to have measurement of airspeed at low speed using inexpensive sensors, so an optional anemometer and a pitot-static probe were employed. The probe provides required performance at an order of magnitude reduction in replacement cost by leveraging a plastic printed housing and low cost encoders and pressure sensors. The use of this probe over other available options also removed the requirement for analogue voltage measurements for the avionics system, a further cost reduction and simplification. Calibration of the probe is planned to be performed using an in-flight, GPS based routine developed at NASA Langley for the AirSTAR system under the Integrated Resilient Aircraft Control (IRAC) project. ${ }^{13}$

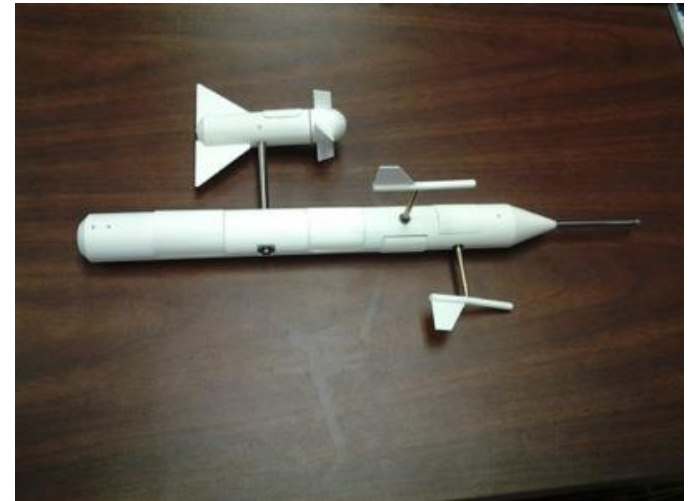

Figure 11. Low cost custom digital airdata probe

\section{Flight Testing Approach}

The GL-10 has an advantage over many other VTOL and conventional takeoff and landing aircraft in that it can be easily tethered from above for VTOL, hover, and some low speed flight testing. This is especially useful considering this aircraft is using prototype avionics. Tethered testing allows the researchers to test the aircraft's power and avionic systems in-flight with very little risk to the airframe. Tethered testing is performed in several different facilities. For very low altitude tethered flight-testing several indoor high bay areas or Langley's Gantry facility is used. At times it is beneficial to fix the aircraft's translational axes and focus on the control systems rotational stabilization or powered lift trim. For that purpose several free-to-pitch, roll, or yaw rigs, as well as a 3axis gimbal rig are available. However, it is often simplest to go straight to hover testing.

One approach used on the project is to engage a skilled RC pilot in the control development process. The pilot is trained on the aircraft type using very inexpensive lightweight aircraft. These aircraft are flown with nothing more than rate gyroscopes for feedback stabilization and simple actuation allocation appropriate for each flight mode. As expected these aircraft are very challenging to pilot, though it is still found that the pilot evaluations of these aircraft are useful to compile lists of abort procedures, the preferred handling qualities, and records of gross aircraft behavior.

The untethered flight test procedure for the 50\% scale GL-10 depends heavily on the results of the $35 \%$ scale aircraft. System and parameters ID maneuvers are to be performed for the low speed portions of the flight envelope for both aircraft. Since the aircraft can trim at multiple angles of attack throughout the transition corridor, several trim variations will be tested with aerodynamic models validated about them. Most of this low speed testing will occur while tethered. For untethered testing, a trim condition will be used with a moderate wing tilt angle that is determined to have large control margin from simulation test results. The aircraft will be flown from the hover mode to the mid-speed trim condition to perform more system identification tests. So, an incremental approach is chosen 
for envelope expansion. This approach has been common for VTOL aircraft and was employed on the XV-15 among others. ${ }^{16}$ After these tests and subsequent simulation refinements, a trajectory will be chosen that maximizes excess control power through transition. The aircraft once flown through outbound transition will perform system ID tests around the cruise flight mode. The aircraft returns to hover initially using a zoom-climb maneuver or other trajectory determined to have large excess control power available to the pilot. After further analysis, a more benign transition maneuver for the inbound transition from cruise to hover will be designed and performed, thus completing the primary project objective.

\section{Summary}

Ground work for the GL-10 project completed to date includes completion of a DOE wind tunnel test, development of an aerodynamic model using GL-10 tunnel test results and propeller test data, derivation and implementation of the vehicle equations of motion into simulation, and inclusion of the aerodynamic models into that simulation. Multiple prototype aircraft have been fabricated at approximately the $15 \%, 35 \%$, and $50 \%$ scales. Some of the small scale aircraft have been flown in an effort to train a skilled RC pilot in the aircraft type. This paper described an approach proposed to complete the primary objective of the project, a piloted demonstration of LEVTOL with inbound and outbound transitions to and from the conventional airplane modes. This proposed approach includes using L1 adaptive control architecture, a control allocation study and design, trajectory optimization and analysis, in-flight system identification, and a incremental flight-test buildup approach resulting in completing the projects primary objective, a remotely piloted demonstration of the vehicle's basic capabilities. Using this approach, the project will have in hand the data, tools, and avionics required to complete the more ambitious secondary objectives of automatic navigation including autonomous takeoff and landing at unimproved sites.

\section{References}

${ }^{1}$ Benefits of Hybrid-Electric Propulsion to Achieve 4x Cruise Efficiency for a VTOL UAV, William J. Fredericks, Mark D. Moore, and Ronald C. Busan, 2013 International Powered Lift Conference. August.

${ }^{2}$ Lieblein, S., et al., "Exploring in Aeronautics", NASA EP-89, 1971, pp. 238, 211-245.

${ }^{3}$ Busan, R., Rothhaar, P., Croom, M., Murphy, P., Grafton, S. O-Neal, A., "Enabling Advanced Wind-Tunnel Research Methods Using the NASA Langley 12-Foot Low Speed Tunnel” AIAA AVIATION 2014 Conference, June 2014.

${ }^{4}$ Montgomery, Douglas, C., "Design and Analysis of Experiments," $8^{\text {th }}$ ed.,Wiley, 2013.

${ }^{5}$ Landman, Drew, Simpson, Jim, Vicroy, Dan D., and Parker, Peter, "Response Surface Methods for Efficient Complex Aircraft Configuration Aerodynamic Characterization,” Journal of Aircraft, Vol. 44, No. 4, July-August 2007.

${ }^{6}$ Landman, Drew, Simpson, Jim, Vicroy, Dan D., and Parker, Peter, "Hybrid Design for Aircraft Wind-Tunnel Testing Using Response Surface Methodologies,” Journal of Aircraft, Vol. 44, No. 4, July-August 2007.

${ }^{7}$ Hovakimyan, N., Cao, C., Kharisov, E., Xargay, E. and Gregory, I.M. "L1 Adaptive Control for Safety Critical Systems: Guaranteed robustness with fast adaptation.” IEEE Control Systems Magazine, October 2011.

${ }^{8}$ Davidson, J.B., Lallman, F.J., Bundick, W.T., "Real-time adaptive control allocation applied to a high performance aircraft," $5^{\text {th }}$ SIAM Conference on Control and Its Applications, 2001.

${ }^{9}$ Jr1. Klein, V., and Morelli, E., “Aircraft System Identification: Theory and Practice," AIAA Education Series, AIAA, 2006.

${ }^{10} \mathrm{Jr}$. Morelli, E., "Flight-Test Experiment Design for Characterizing Stability and Control of Hypersonic Vehicles," Journal of Guidance, Control, and Dynamics, Vol. 32, No. 3, May-June 2009, pp. 949-959.

${ }^{11}$ Jr3. Grauer, J., and Morelli, E., “A Method for Real-Time Frequency Response and Uncertainty Estimation," Journal of Guidance, Control, and Dynamics, Vol. 37, No. 1, January-February, 2014, pp. 336-344, electronically published 10 July 2013.

${ }^{12} \mathrm{Jr}$. Morelli, E., " Global Nonlinear Aerodynamic Modeling Using Multivariate Orthogonal Functions," Journal of Aircraft, Vol. 32, No. 2, March-April 1995, pp. 270-277.

${ }^{13}$ Foster, J., and Cunningham, K. "A GPS-Based Pitot-Static Calibration Method Using Global Output-Error Optimization," AIAA Aerospace Sciences Meeting, January 2010.

${ }^{14}$ Tosti, L. P., "Longitudinal Stability and Control of a Tilt-Wing VTOL Aircraft Model With Rigid and Flapping Propeller Blades", NASA Technical Note D-1365, 1962.

${ }^{15}$ Kelley, H. L., Reeder, J. P., Champine, R. A., "Summary of a Flight-Test Evaluation of the CL-84 Tilt-Wing V/STOL Aircraft”, NASA TM X-1914, 1970

${ }^{16}$ Maisel, M. D., Giulianetti, D. J., Dugan, D.C., “The History of the XV-15 Tilt Rotor Research Aircraft: From Concept to Flight”, NASA SP-2000-4517, 2000. 